\title{
In-medium effects and dilepton emission in heavy-ion collisions at SIS energies
}

\section{M.D. Cozma ${ }^{\star a}{ }^{\dagger}$ E. Santini ${ }^{b}$ C. Fuchs ${ }^{b}$ M.V. Martemyanov ${ }^{b, c}$ M.I. Krivoruchenko ${ }^{b, c}$ and} A. Faessler ${ }^{b}$

${ }^{a}$ National Institute for Physics and Nuclear Engineering Atomiştilor 407, 077125 Măgurele-Bucharest, Romania

${ }^{b}$ Institut für Theoretische Physik, Universität Tübingen Auf der Morgenstelle 14, 72076 Tübingen, Germany

${ }^{c}$ Institute for Theoretical and Experimental Physics

B. Cheremushkinskaya 25, 117259 Moscow, Russia

E-mail: Cozma@niham.nipne.ro

\begin{abstract}
Dileptons represent a unique probe for nuclear matter under extreme conditions reached in heavyion collisions. They allow the study of meson properties, like mass and decay width, at various density and temperature regimes. We use the extended vector meson dominance (eVMD) model developed in Tübingen to determine the in-medium properties of the $\rho$ and $\omega$ vector mesons by computing their in-medium spectral functions. Contributions to the in-medium self-energies due to excitation of nucleonic resonances are supplemented by non-resonant Compton scattering and $\sigma$ meson exchange terms. Dilepton spectra for $\mathrm{C}+\mathrm{C}$ heavy-ion collisions at 1.0 and $2.0 \mathrm{AGeV}$ within spectral function approach and the traditional Brown-Rho scalling/collisional broadening scenario are computed and compared. Finally, a preliminary comparison with the available HADES collaboration experimental data for $\mathrm{C}+\mathrm{C}$ at $2.0 \mathrm{AGeV}$ is presented, revealing an important discrepancy in the $0.2-0.7 \mathrm{GeV}$ invariant mass region.
\end{abstract}

PACS: $12.40 \mathrm{Vv}, 25.75 .-\mathrm{q}, 25.75 \mathrm{Dw}$

Critical Point and Onset of Deconfinement - 4th International Workshop

July 9 - 13, 2007

Darmstadt, Germany

\footnotetext{
* Speaker.

${ }^{\dagger}$ Work performed during stay at ITP, Universität Tübingen, Germany.
} 


\section{Introduction}

Dileptons produced in heavy ion reactions present an unique opportunity for the study of nuclear matter under extreme conditions, providing a clear view on effective degrees of freedom at high baryon density and temperature. It has been argued that their differential spectra could reveal information about chiral restoration and in-medium properties of hadrons [1, 2, 3]. Theoretically, there exists an abundance of models that predict a change of vector meson masses and widths in high density/temperature nuclear matter: Brown-Rho scaling [1] is equivalent with a decrease of vector meson masses in nuclear medium; models based on QCD sum rules [⿰] - and effective hadronic models [4, 8, 6, 7, 8, 9] reach similar conclusions.

Experimentally, a significant enhancement of the dilepton spectrum in low energy heavy ion collisions [10, 11] over the known hadronic sources has been observed. This could not be explained by present models, as opposed to the ultrarelativistic case, even when the in-medium spectral functions or the dropping mass scenarios are taken into account [12, 13]. Other scenarios like contributions from quark-gluon plasma, in-medium modifications of the $\eta$ mass or decoherence effects [14] have proved only partially successful. Recent results [15] on dimuon spectra with high resolution in the vicinity of the $\rho$ - $\omega$ peak seem to rule out a naive dropping mass scenario but support the picture of modified $\rho-\omega$ spectral functions. The HADES collaboration at GSI is dedicated to the experimental measurement of dilepton spectra with unprecedented mass resolution for the reactions $\mathrm{A}+\mathrm{A}, \mathrm{p}+\mathrm{A}$ and $\pi+\mathrm{A}[16]$. Recently, results for $\mathrm{C}+\mathrm{C}$ collisions at $2.0 \mathrm{AGeV}$ have been published [17] and preliminary results for $\mathrm{C}+\mathrm{C}$ at $1.0 \mathrm{AGeV}$ and $\mathrm{p}+\mathrm{p}$ and $\mathrm{p}+\mathrm{d}$ at $1.25 \mathrm{GeV}$ have been announced. This wealth of experimental information will hopefully allow the extraction of in-medium properties of $\rho$ and $\omega$ vector mesons.

In this proceeding we present a recent development of our model for dilepton emission in heavy-ion reactions and present results for $\mathrm{C}+\mathrm{C}$ collisions at 1.0 and $2.0 \mathrm{AGeV}$ together with a preliminary comparison with the HADES data for $\mathrm{C}+\mathrm{C}$ at $2.0 \mathrm{AGeV}$. Vector meson and dilepton production are described within the framework of the resonance model developed in [18, 19, 20] in combination with the relativistic quantum molecular dynamics (RQMD) transport model for heavy ion collisions [14] (Section II). In previous works [14, 21] influence of medium effects has been accounted by a phenomenological dropping of meson masses (Brown-Rho scaling) and collisional broadening of meson decay widths together with quantum decoherence effects. In the present work we employ the extended vector meson dominance model (eVMD) [18, 19, 20] to determine medium corrections to vector meson self-energies due to excitation of nucleonic resonances. Additional contributions, such as non-resonant Compton scattering of vector mesons off nucleons and elastic scattering with the exchange of $\sigma$ mesons have also been considered (Section III). In Section IV theoretical dilepton spectra for $\mathrm{C}+\mathrm{C}$ collisions at 1.0 and $2.0 \mathrm{AGeV}$ are presented making use of in-medium description of vector mesons both in the spectral function and the more traditional Brown-Rho/collisional broadening approaches. We conclude with a preliminary comparison of our theoretical dilepton spectrum for $\mathrm{C}+\mathrm{C}$ reaction at $2.0 \mathrm{AGeV}$ with the available $\mathrm{HADES}$ data. 


\section{The model}

\subsection{Elementary dilepton sources}

In the energy region of interest in this paper there are two main classes of processes that lead to dilepton emission: decay of light unflavored mesons and decay of nucleon and $\Delta$ resonances. At incident energies of $1 \mathrm{AGeV}$ the cross-sections for meson production $\mathscr{M}=\eta, \eta^{\prime}, \rho, \omega, \phi$ are small and these mesons do not play an important role in the dynamics of heavy-ion collisions. Their production can thus be treated perturbatively, in contrast to the case of the pion. The decay to a dilepton pair takes place through the emission of a virtual photon. The differential branching ratios for the decay of a meson to a final state $X e^{+} e^{-}$can be written

$$
d B(\mu, M)^{\mathscr{M}, \pi \rightarrow e^{+} e^{-} X}=\frac{d \Gamma(\mu, M)^{\mathscr{M}, \pi \rightarrow e^{+} e^{-} X}}{\Gamma_{\text {tot }}^{\mathscr{M}, \pi}(\mu)},
$$

with $\mu$ the meson mass and $M$ the dilepton mass. Three types of such decays have been considered: direct decays $\mathscr{M} \rightarrow e^{+} e^{-}$, Dalitz decays $\mathscr{M} \rightarrow \gamma e^{+} e^{-}, \mathscr{M} \rightarrow \pi(\eta) e^{+} e^{-}$and four-body decays $\mathscr{M} \rightarrow \pi \pi e^{+} e^{-}[18]$. The decay channels there are most important quantitatively for heavy-ion collisions at 1 and $2 \mathrm{GeV} /$ nucleon being $\pi^{0} \rightarrow \gamma e^{+} e^{-}$and $\eta \rightarrow \gamma e^{+} e^{-}$.

For the description of decays of baryonic resonances into dileptons an extension of the vector meson dominance (VMD) model has been employed [18, 19]. The original VMD model assumes that decays of baryon resonances run through an intermediate virtual meson ( $\rho$ or $\omega)$ required for the description of the form-factors entering in the calculation of the radiative $(R \rightarrow N \gamma)$ and mesonic $(R \rightarrow N V)$ decays. Such a model does not allow the simultaneous description of both radiative and mesonic decays [20, 22]. Furthermore the quark counting rules require a stronger suppression of the transition form-factor than the $1 / t$ behavior predicted by the naive VMD. Similarly the $\omega \pi \gamma$ transition form-factor shows an asymptotic $1 / t^{2}$ behavior [23]. An extension of the VMD to allow contributions from radially excited vector mesons ( $\rho(1250), \rho(1450), \ldots$ in Ref. [19]) that interfere destructively with the ground state vector mesons ( $\rho$ in this example) allow for a resolution of the mentioned problems of the original VMD and describe the radiative and mesonic decays in a unitary way.

In terms of the branching ratios for the Dalitz decays of the baryon resonances, the cross section for $e^{+} e^{-}$production from the initial state $X^{\prime}$ together with the final state $N X$ can be written

$$
\begin{gathered}
\frac{d \sigma(s, M)^{X^{\prime} \rightarrow N X e^{+} e^{-}}}{d M^{2}}=\sum_{R} \int_{\left(m_{N}+M\right)^{2}}^{\left.\sqrt{s}-m_{X}\right)^{2}} d \mu^{2} \\
\times \frac{d \sigma(s, \mu)^{X^{\prime} \rightarrow R X}}{d \mu^{2}} \sum_{V} \frac{d B(\mu, M)^{R \rightarrow V N \rightarrow N e^{+} e^{-}}}{d M^{2}},
\end{gathered}
$$

where $\mu$ is the mass of the baryon resonance $R$ which has the production cross-section $d \sigma(s, \mu)^{X^{\prime} \rightarrow X R}$ and $d B(\mu, M)^{R \rightarrow V N \rightarrow e^{+} e^{-}}$being the differential branching ratio for the decay of the resonance $R \rightarrow N e^{+} e^{-}$through the vector meson $V$. The initial state $X^{\prime}$ could consist of two baryons $\left(X^{\prime}=\right.$ $\left.N N, N R, R R^{\prime}\right)$ or of one nucleon and a pion $(X=\pi N)$. The dilepton decay rate can be found, once the width $\Gamma\left(R \rightarrow N \gamma^{*}\right)$ is known by using the factorization prescription

$$
d \Gamma\left(R \rightarrow N e^{+} e^{-}\right)=\Gamma\left(R \rightarrow N \gamma^{*}\right) M \Gamma\left(\gamma^{*} \rightarrow e^{+} e^{-}\right) \frac{d M^{2}}{\pi M^{4}},
$$


with

$$
M \Gamma\left(\gamma^{*} \rightarrow e^{+} e^{-}\right)=\frac{\alpha}{3}\left(M^{2}+2 m_{e}^{2}\right) \sqrt{1-\frac{4 m_{e}^{2}}{M^{2}}} .
$$

The decay width $\Gamma\left(R \rightarrow N \gamma^{*}\right)$ is described within the extended VMD model [19] in terms of three transition form-factors (magnetic, electric and Coulomb) in case of a resonance with spin $J>1 / 2$ and two for $J=1 / 2$, which is just the number of independent helicity amplitudes for the respective spin value. The free parameters of the model are fixed by constraining the asymptotic form of the form-factors by quark counting rules [24] and fitting to the experimental data for photo-production and electro-production amplitudes and partial-wave analysis for multichannel $\pi N$ scattering. A consistent description of radiative and mesonic decays could be achieved. Further details about the eVMD, including the decoherence effect, can be found in Ref. [19].

\section{Vector mesons in nuclear medium}

Properties of elementary particles in vacuum as well as in a nuclear medium are encoded in the two-point function $\left\langle\Omega\left|T\left(V_{\mu}(x) V_{v}(y)\right)\right| \Omega\right\rangle$ also known as the propagator. One starts from the expression of the free-propagator, which in the case of a spin- 1 particle ( $\rho$ or $\omega$ vector meson) can be put in the form

$$
D_{\mu v}^{0}(k)=\frac{-g_{\mu v}+k_{\mu} k_{v} / k^{2}}{k^{2}-m^{2}+i \varepsilon}+\frac{1}{m^{2}} \frac{k_{\mu} k_{v}}{k^{2}} .
$$

Due to self-interactions and scattering off particles in a dense medium the free propagator is modified, the two-point function receives higher order contributions which are generically accounted for in the self-energy $\Sigma_{\mu \nu}$. Making use of Lorentz covariance the most general expression for the self-energy reads

$$
\Sigma_{\mu v}(k, n)=g_{\mu \nu} \Sigma_{1}(k, n)+k_{\mu} k_{v} \Sigma_{2}(k, n)+n_{\mu} n_{v} \Sigma_{3}(k, n)+k_{\mu} n_{v} \Sigma_{4}(k, n),
$$

which in the special case of gauge invariant interactions can be simplified to

$$
\begin{aligned}
& \text { in vacuum : } \Sigma_{\mu v}=P_{\mu v}^{T}(k) \Sigma_{v a c}(k) \\
& \text { in medium : } \Sigma_{\mu v}=T_{\mu v} \Sigma^{T}(k)+L_{\mu \nu} \Sigma^{L}(k) .
\end{aligned}
$$

Here the $P_{\mu \nu}^{T}, T_{\mu \nu}$ and $L_{\mu v}$ are projectors on the four-dimensional transverse, three-dimensional transverse and respectively longitudinal degrees of freedom whose expressions can be found elsewhere. The four-vector $n_{\mu}$ characterizes the properties of nuclear matter in an arbitrary rest-frame and reduces to $\left(m_{N}, \overrightarrow{0}\right)$ in its rest frame. The above expression for the self-energy allows the summation of the perturbative solution of the Dyson-Schwinger equation for the spin-1 propagator, to arrive at the final expression

$$
D_{\mu v}(k)=-\frac{L_{\mu v}(k)}{k^{2}-m^{2}-\Sigma^{L}\left(k^{2}\right)}-\frac{T_{\mu v}(k)}{k^{2}-m^{2}-\Sigma^{T}\left(k^{2}\right)}+\frac{1}{m^{2}} \frac{k_{\mu} k v}{k^{2}} .
$$

The spectral function, defined as the imaginary part of the propagator, contains all the relevant information about particle properties, such as pole mass and decay widths, in nuclear matter. For 


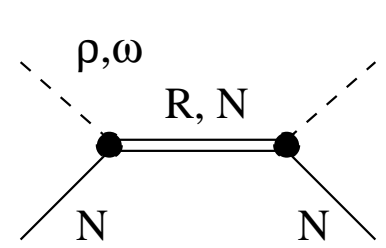

a)

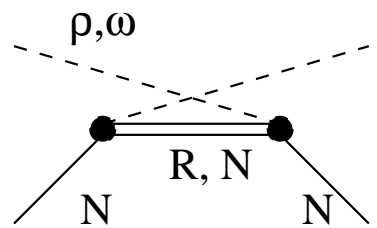

b)

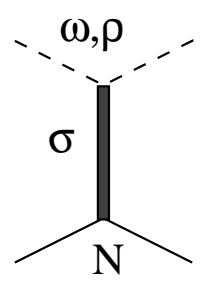

c)

Figure 1: Diagrammatic representation of processes contributing to the in-medium vector meson selfenergies: a) direct Compton and b) crossed Compton scattering of mesons on nucleons with (R) and without $(\mathrm{N})$ excitation of nucleonic resonances; c) scattering of vector mesons off nucleons with the exchange of a $\sigma$ mesons.

the case of spin-1 mesons, due to the fact that manifest Lorentz covariance is broken in nuclear medium, one has to deal with two such spectral functions characterizing separately the longitudinal and transversal degrees of freedom:

$$
A^{L, T}(k, n)=-\frac{1}{\pi} \frac{-\mathscr{I} m \Sigma^{L, T}(k, n)}{\left(k^{2}-m_{V}^{2}-\mathscr{R} e \Sigma^{L, T}(k, n)\right)^{2}+\left(-\mathscr{I} m \Sigma^{L, T}(k, n)\right)^{2}} .
$$

Eventhough not stated explicitly, self-energies, and implicitly also spectral functions, depend independently on $k^{0}$ and $\vec{k}$ at finite nuclear matter densities as effect of the finite Fermi momentum $p_{F}$. The longitudinal and transversal part of the meson self-energies each can be written as the sum of a vacuum term and a medium contribution: $\Sigma^{L, T}(k, n)=\Sigma_{\text {vac }}^{L, T}(k, n)+\Sigma_{\text {med }}^{L, T}(k, n)$. The vacuum contribution is conventionally defined as being purely imaginary, the real part being implicitly absorbed in the mass term that defines the pole mass $m_{V}$ of the meson in vacuum, $\Sigma_{v a c}^{L, T}(k, n)=-i \sqrt{k^{2}} \Gamma_{v a c}$ (see [25] for the energy dependence parametrization of vacuum decay widths of $\rho$ and $\omega$ vector mesons).

In our model, a few contributions to vector mesons self-energies in nuclear matter have been considered. First we discuss contributions due to excitation of nucleon resonances. For simplicity we will consider a schematic model of a spin $1 / 2$ resonance which is excited by the absorption of a vector meson (of isospin 0) by a nucleon: $\mathscr{L}_{\text {int }}=-g \bar{\psi}_{R} \Gamma^{\mu} \psi V_{\mu}$. There are two contributions to the self-energy tensor originating from the direct and crossed contributions to the Compton scattering of mesons off nucleons (Fig. (1)a)-b)):

$$
\begin{aligned}
\Sigma_{\mu \nu}^{R(\text { direct })} & =\int_{|\vec{p}|<p_{F}} d^{3} \vec{p} \operatorname{Tr}\left[\frac{p+m_{N}}{2 m_{N}} \Gamma_{\mu}^{*} \frac{\not p+\not k+m_{R}}{(p+k)^{2}-m_{R}^{2}+i m_{R} \Gamma_{R}} \Gamma_{\nu} u(p)\right], \\
\Sigma_{\mu \nu}^{R(\text { crossed })} & =\int_{|\vec{p}|<p_{F}} d^{3} \vec{p} \operatorname{Tr}\left[\frac{p+m_{N}}{2 m_{N}} \Gamma_{\nu}^{*} \frac{\not p-\not k+m_{R}}{(p-k)^{2}-m_{R}^{2}+i m_{R} \Gamma_{R}} \Gamma_{\mu} u(p)\right] .
\end{aligned}
$$

where at first order in a density expansion, nucleon and resonance propagators are unaffected by the presence of the dense nuclear medium. The parameters $m_{R}$ and $\Gamma_{R}$ stand for the mass and respectively decay width of the nucleonic resonance considered, the latter being an energy dependent quantity. In the actual calculations, to determine $\Sigma^{\mu v}$ for a particular resonance R, the matrix elements of the RNV vertex provided by the eVMD model described in the previous Section have been used. For the calculation of the dilepton yield in heavy-ion collisions a meson self-energy averaged over the transversal and longitudinal degrees of freedom will employed, 
$\Sigma(k, n)=\left(2 \Sigma^{T}(\mathrm{k}, \mathrm{n})+\Sigma^{L}(\mathrm{k}, \mathrm{n})\right) / 3$, which can be used in Eq. (3.5) instead of $\Sigma^{L, T}$ to obtain an average spectral function $A\left(k_{0}, \vec{k}\right)$.

Similar expressions to Eq. (3.7) are obtained for the contributions due to non-resonant Compton scattering of vector mesons off nucleons (see Fig. (1)a)-b)). The couplings of $\rho$ and $\omega$ vector mesons to nucleons were chosen to be

$$
\begin{aligned}
& \mathscr{L}_{N N \rho}=g_{\rho}^{V} \bar{\psi} \gamma^{\mu} \vec{\tau} \psi \cdot \vec{\rho}_{\mu}+\frac{i g_{\rho}^{T}}{4 m_{N}} \bar{\psi} \sigma^{\mu v} \vec{\tau} \psi \cdot\left(\partial_{\mu} \vec{\rho}_{v}-\partial_{v} \vec{\rho}_{\mu}\right), \\
& \mathscr{L}_{N N \omega}=g_{\omega}^{V} \bar{\psi} \gamma^{\mu} \psi \omega_{\mu},
\end{aligned}
$$

the values of the coupling constants reproducing the ones used in the Bonn OBE model for the NN interaction [26]: $g_{\rho}^{V 2} / 4 \pi=0.84, g_{\rho}^{T} / g_{\rho}^{V}=6.09$ and $g_{\omega}^{V 2} / 4 \pi=20.00$. The only difference with expressions in Eq. (3.7) resides in the fact that each of the contribution is now multiplied with a step function: $\theta\left(|\vec{p}+\vec{k}|-p_{F}\right)$ and respectively $\theta\left(|\vec{p}-\vec{k}|-p_{F}\right)$ as a result of Pauli blocking of the scattered on nucleons. As an effect, the sum of the two contributions is not gauge invariant anymore, as opposed to the vacuum case. The third and last in-medium contribution to the vector mesons self-energies is due to scattering of mesons off nucleons with the exchange of $\sigma$ mesons as in Fig. (1)c). The Bonn $\sigma N N$ interaction with $g_{\sigma}^{2} / 4 \pi=6.73$ is used, while the $\rho \rho \sigma$ vertex is described by the effective Lagrangian

$$
\mathscr{L}_{\rho \rho \sigma}=-g_{\rho \rho \sigma}\left(\partial_{\mu} \vec{\rho}_{v}-\partial_{v} \vec{\rho}_{\mu}\right) \cdot\left(\partial^{\mu} \vec{\rho}^{v}-\partial^{v} \vec{\rho}^{\mu}\right) \sigma
$$

The coupling constant $g_{\rho \rho \sigma}$ is determined by requiring that the value of the branching ratio $B\left(\rho^{0} \rightarrow\right.$ $\left.\pi^{+} \pi^{-} \pi^{+} \pi^{-}\right)=(1.8 \pm 0.9) \times 10^{-5}$ is reproduced supposing that the mentioned decay is a two-step process: $\rho^{0} \rightarrow \rho^{0} \sigma \rightarrow \pi^{+} \pi^{-} \pi^{+} \pi^{-}$. A similar interaction is supposed to exist for the $\omega$ meson with $g_{\omega \omega \sigma}=3 g_{\rho \rho \sigma}$.

We turn now to quantitative results for the spectral functions of the $\rho$ and $\omega$ vector mesons. In obtaining the present results the following set of nucleonic resonances has been considered: $N^{*}(1440), N^{*}(1520), N^{*}(1535), N^{*}(1650), N^{*}(1680), \Delta^{*}(1232), \Delta^{*}(1620), \Delta^{*}(1700)$ and $\Delta^{*}(1905)$. In Fig. (2) we present results for the $\rho$ meson average spectral function $A\left(k_{0}, \vec{k}\right)$ introduced before for two values of the meson three-momentum $k$ : $\mathrm{k}=0.0 \mathrm{GeV}$ which corresponds to a meson at rest with respect to nuclear matter and $\mathrm{k}=0.5 \mathrm{GeV}$. In the upper plots results at various nuclear matter densities: $1 / 2 \rho_{0}, \rho_{0}, 2 \rho_{0}$ are presented by including only contributions from nucleonic resonances to the in-medium self-energies and compared with vacuum. It is readily visible that with the increase of density the original Breit-Wigner peak gets broader and shifted towards higher invariant masses, an effect mainly due to contributions from the $\Delta$ resonances with a mass higher than 1600 $\mathrm{MeV}$. In-medium contributions are most readily visible in a secondary peak at invariant masses around $0.5 \mathrm{GeV}$ or below, originating from the excitation of $\mathrm{N}^{*}(1520), \mathrm{N}^{*}(1535)$, and $\Delta(1620)$. Its magnitude increases with increasing density, as one would expect from the low density theorem and displays a strong dependence on the value of meson's three-momentum. The hadronic models of Post et al.[22] and Lutz et al.[8] provide a similar picture, up to a quantitative agreement with the former model due to a similarity in both the approach and included physics. The latter model relies on a dynamic generation of nucleonic resonances within a relativistic and unitary approach to pion and photo-nucleon reactions and as a result the decay width of the $\mathrm{N}^{*}(1520)$ resonance 


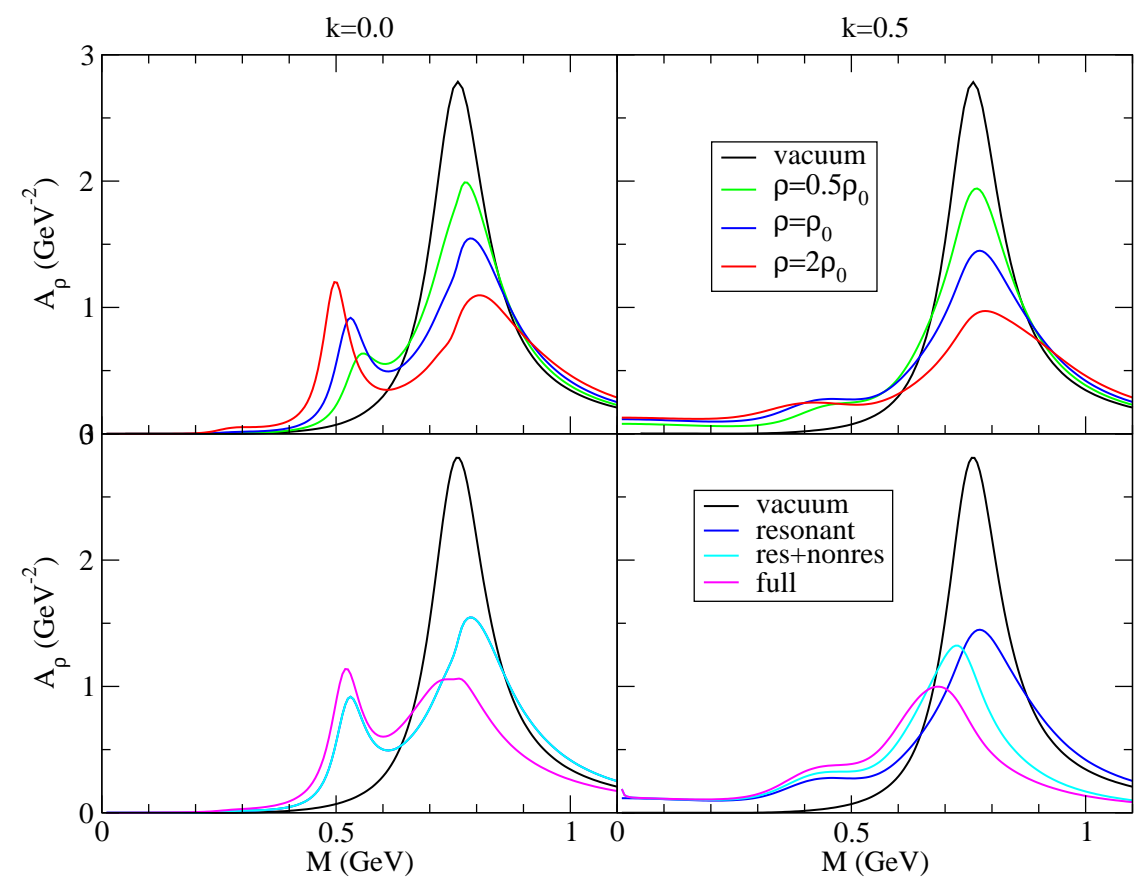

Figure 2: Averaged spectral functions of the $\rho$ vector meson for two values of the three-momentum $\mathrm{k}$ : $\mathrm{k}=0.0$ $\mathrm{GeV}$ (left panels) and respectively $\mathrm{k}=0.5 \mathrm{GeV}$ (right panels). In the upper panels the $\rho$ spectral function in nuclear medium with resonant contributions only are displayed for different values of the density parameter. In the lower panel spectral functions are plotted by taking into account progressively the various in-medium contributions discussed in the text at saturation density of nuclear matter. The vacuum spectral functions are also plotted for comparison.

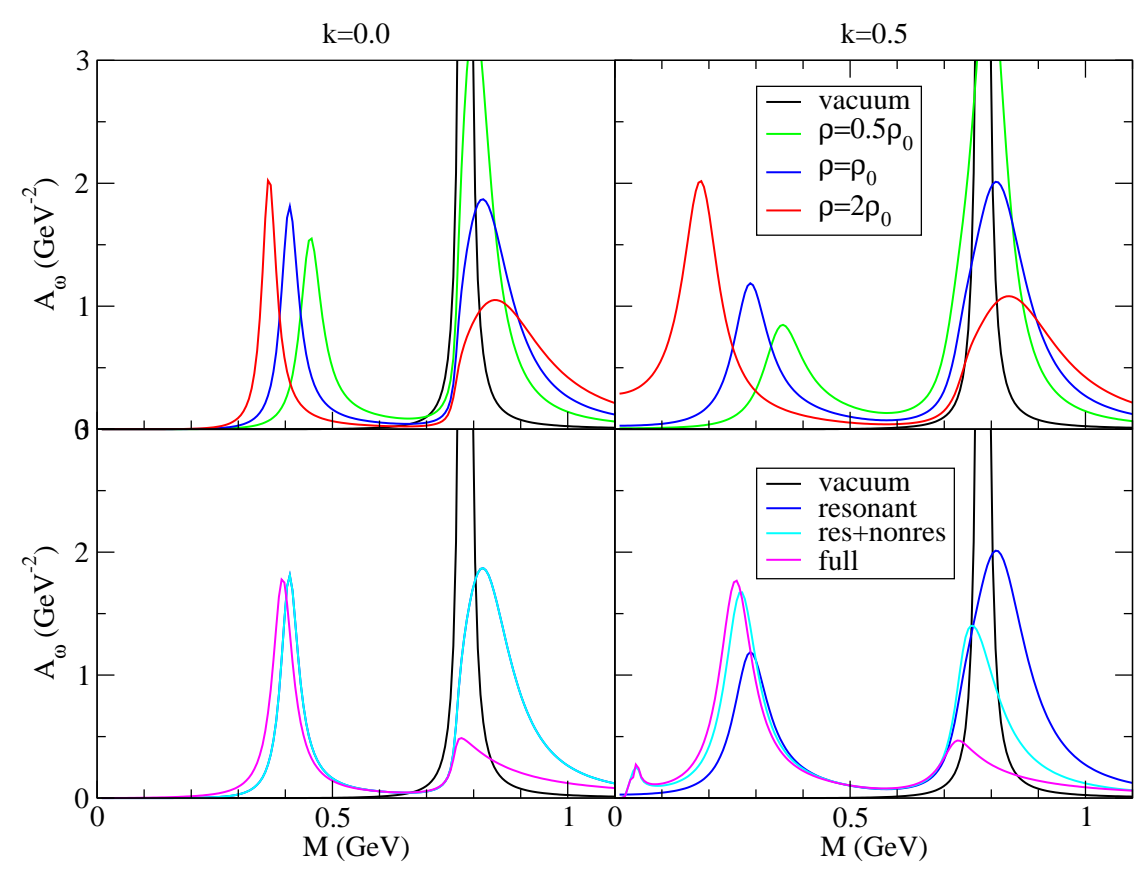

Figure 3: The same as in Fig. 2 but for the $\omega$ meson. 
is roughly an order of magnitude smaller than the listed PDG vacuum value having as a result a rather small impact of nucleonic resonances on the vector meson spectral functions at small invariant masses. In the lower part of Fig. (2) the importance of the non-resonant contributions to in-medium spectral functions at nuclear-matter saturation density is studied by adding these terms progressively to the final result. The non-resonant Compton scattering contribution increases with the meson three-momentum leading to a shift of the original meson branch towards lower invariant masses and increasing the decay width somewhat. Inclusion of $\sigma$ meson exchange terms leads to a similar effect.

In a subsequent figure, Fig. (3), our results for the $\omega$ meson are presented in an identical display as for the $\rho$ meson. A strong influence of resonant contributions is observed, already at densities well below the nuclear matter saturation density with results in the appearance of a sharp $(30 \div 100 \mathrm{MeV}$ ) second branch in-medium at invariant masses of less than $0.5 \mathrm{GeV}$. This is mainly due to excitation of $\mathrm{N}^{*}(1535)$ and $\mathrm{N}^{*}(1520)$ in the order of importance. The original peak shifts towards slightly higher masses with a decay width of about an order of magnitude higher than in vacuum and strength comparable to the newly observed second branch. Qualitatively the models of Post et al. [22] and Lutz et al. [8] agree with our findings, with the exception the strength of second $\omega$ branch is considerably smaller relatively to the original peak. The inclusion of the non-resonant terms has as main effect a a shift of the original $\omega$ peak towards smaller masses supplemented by a strong suppression. Consequently, in nuclear medium, the $\omega$ vector meson survives as a quasiparticle of lower mass than in vacuum $(300 \div 400 \mathrm{MeV})$ due to excitation of nucleonic resonances.

\section{Dilepton spectrum}

In this Section we provide theoretical predictions for dilepton emission in $\mathrm{C}+\mathrm{C}$ collisions at 1 and $2 \mathrm{AGeV}$. The main purpose will be to compare previous calculations that include in-medium effects in a more traditional way, i.e. via Brown-Rho scaling of the vector meson masses and empirical collisional broadening of the decay widths, with preliminary results obtained using $\rho$ and $\omega$ mesons described by the in-medium spectral functions of the previous Section. Our results are shown in Fig. (4).

Dilepton spectra at intermediate energies (probed by DLS and HADES experiments) are more sensitive to the $\omega$ meson collisional broadening. In absence of in-medium modifications of mesons the invariant mass dilepton spectrum would show a pronounced $\omega$ peak (black curve in Fig. (4)). In the DLS experiment such an enhancement has not been observed. Despite the limited mass resolution in [14] an in-medium $\omega$ width of $\Gamma_{\omega}^{t o t}=100 \div 300 \mathrm{MeV}$ at nuclear matter density $\rho=$ $1.5 \rho_{0}$ has been extracted from the DLS data. The modification of the $\rho$ width was found to be similar in magnitude, i.e. $\Gamma_{\rho}^{\text {tot }}=200 \div 300 \mathrm{MeV}$ (again at $\rho=1.5 \rho_{0}$ ).

For the Brown-Rho scaling/collisional broadening scenario we present calculations with an assumed linear dependence of the $\rho$ and $\omega$ decay widths, i.e. $\Gamma_{V}^{\mathrm{tot}}=\Gamma_{V}^{\mathrm{vac}}+\rho / \rho_{0} \Gamma_{V}^{\mathrm{coll}}$, with $\Gamma_{\omega}^{\mathrm{tot}}=125$ $\mathrm{MeV}, \Gamma_{\rho}^{\mathrm{tot}}=250 \mathrm{MeV}$ at $\rho=\rho_{0}$. Simultaneously, the masses of mesons are supposed to vary as a function of the nuclear matter density at the point where the resonance decay occurs, following a Brown-Rho scaling law $m_{V}^{*}=m_{V}\left(1-\alpha \rho / \rho_{0}\right)$ with $\rho$ the local baryon density and $\alpha=0.2$. Results are indicated by the red curve in Fig. (\$). This calculation also includes contributions of an additional in-medium effect only briefly mentioned in this proceeding, decoherence, which has 


\section{$\mathrm{C}+\mathrm{C} @ 1.0 \mathrm{~A} \mathrm{GeV}$}

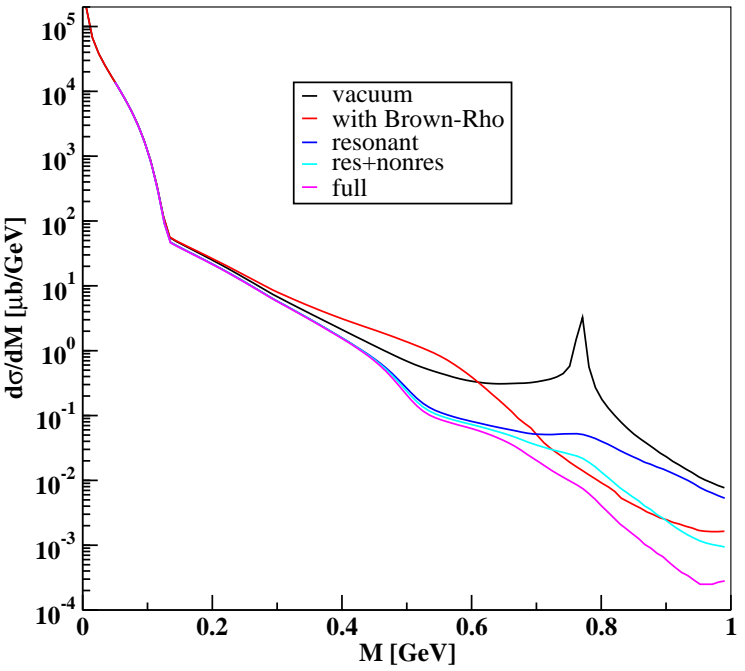

$\mathrm{C}+\mathrm{C} @ 2.0 \mathrm{~A} \mathrm{GeV}$

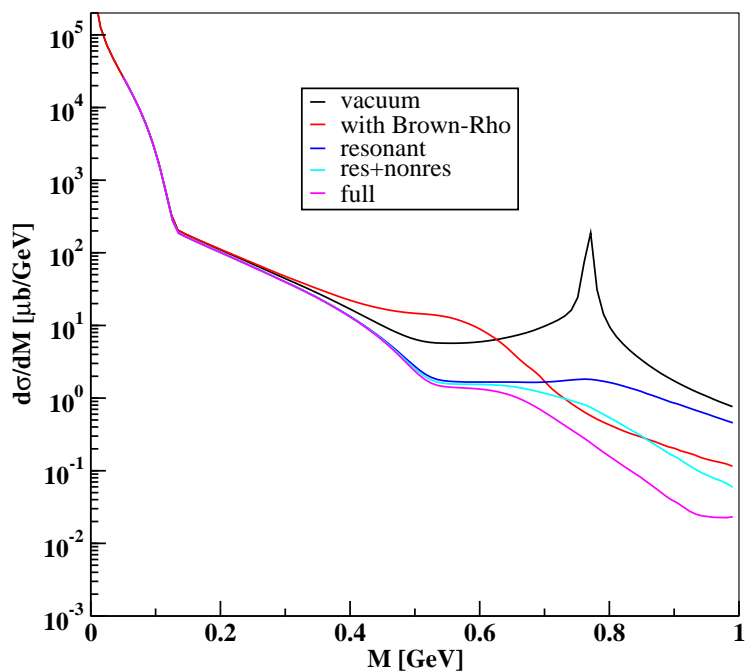

Figure 4: Dilepton spectrum in $\mathrm{C}+\mathrm{C}$ at $1.0 \mathrm{AGeV}$ (left panel) and $2.0 \mathrm{AGeV}$ (right panel). Brown-Rho scenario calculations are depicted by the red curve. Three scenarios for the spectral function approach are presented which differ with respect to what contributions are considered for the computation of the inmedium meson self-energies: only nucleonic resonances (blue curve), nucleonic resonances+non-resonant Compton scattering (cyan curve) and the full model (magenta curve). The vacuum result (black curve) is also shown for comparison.

as a result an increase of the dilepton production cross-section by roughly $\% 50$ at intermediate masses [21]. Sensitivity of the dilepton spectrum with respect to variations of the $\rho$ and $\omega$ collisional widths is mild [21]: variation of $\Gamma_{\rho}^{\mathrm{tot}}=150 \div 250 \mathrm{MeV}$ leads to a modification of the dilepton yield by a factor of 2 in the dilepton mass range $0.5-0.8 \mathrm{GeV}$, while a calculation with a moderately low value for the $\omega$ widths $\Gamma_{\omega}^{\mathrm{tot}}=60 \mathrm{MeV}$ allows for at most $\% 50$ increase in the same mass region.

Medium effects can enter in a few places in the expression for the dilepton production rate Eq. (2.2) and Eq. (2.3). As the decay of a resonance into a dilepton pair runs through an intermediate vector meson (within the VMD approach) the branching ratio $d B(\mu, M)^{R \rightarrow V N \rightarrow N e^{+} e^{-}} / d M^{2}$ is expected to alter. Medium modification will affect both $d \Gamma(\mu, M)^{R \rightarrow V N \rightarrow N e^{+} e^{-}} / d M^{2}$ and $\Gamma(\mu)^{R \rightarrow X}$. The former is accounted for by including in the expression of the vector mesons propagators the effect of the in-medium self-energies. This procedure only affects the ground state $\rho$ and $\omega$ vector mesons, their excited states are supposed to be, in a first approximation, unaffected by the presence of the dense nuclear medium. In-medium modifications of the total decay widths of nucleonic resonances occur due to in-medium modifications of the vector mesons and Pauli blocking of nucleons. At present we have only partially accounted for the first source, i.e. the meson spectral functions with the provision that the total resonance decay widths have not been iterated (as they depend upon each other) to obtain self-consistency.

We present in Fig. (4) theoretical predictions for the dilepton spectrum within the spectral function approach to in-medium vector mesons for three scenarios of in-medium effects: first we only include contributions from nucleonic resonances to in-medium meson self-energies (blue curve) and than gradually add non-resonant Compton scattering (cyan curve) and finally also $\sigma$ meson 


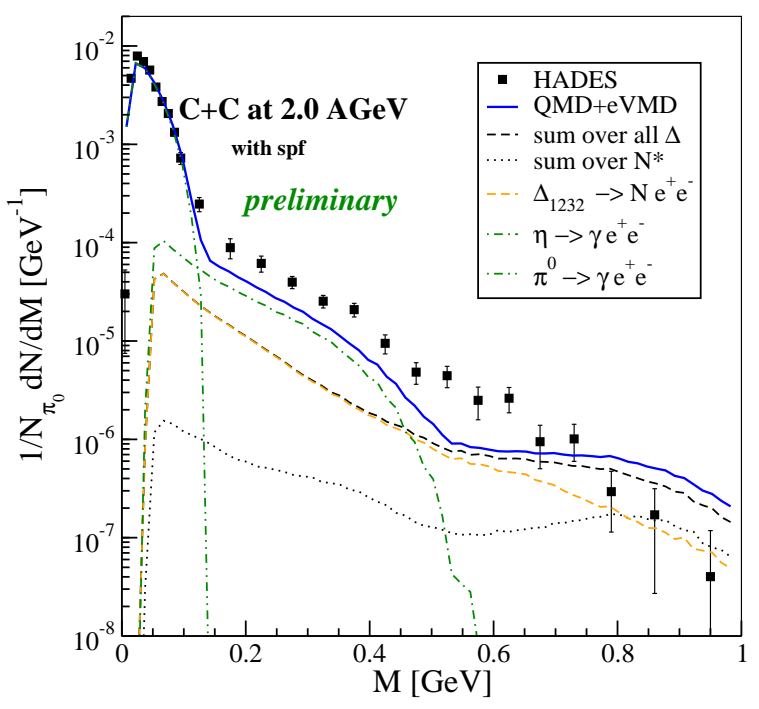

Figure 5: Comparison between the theoretical dilepton spectrum and the experimental result obtained by the HADES collaboration in $\mathrm{C}+\mathrm{C}$ collisions at 2.0 AGeV. Individual contributions of some dilepton emission channels are also shown.

exchange contributions (magenta curve). For these curves the emission of dileptons due to Dalitz decays of pions and $\eta$ mesons does not change. Substantial difference with respect to the traditional Brown-Rho/collisional approach is observed over the whole mass range. At invariant masses below $0.7 \mathrm{GeV}$ the dilepton production rate has decreased by a factor up to 10 due to a strong decrease of the dilepton emission through decays of nucleonic resonances in this mass region, most notably the $\Delta(1232)$ isobar. The effect is more pronounced for the $2 \mathrm{AGeV} \mathrm{C+C}$ reaction, since previously the Dalitz $\eta$ and nucleonic decays were of comparable magnitude, while for the $1 \mathrm{AGeV}$ case the latter dominates. At invariant masses above $0.7 \mathrm{GeV}$ the outcome of the three consired in-medium scenarios vary widely. Inclusion of only nucleonic resonance contribution leads to an important increase of the dilepton cross-section in this region with respect to the old case, while the inclusion of non-resonant background suppresses the dilepton spectrum quite strongly.

In the past year, dilepton spectrum measurements in the $\mathrm{C}+\mathrm{C}$ heavy-ion reactions at $2.0 \mathrm{AGeV}$ and more recently $1.0 \mathrm{AGeV}$ preliminary results due to the HADES collaboration at GSI have been published. Also recently the HADES acceptance filter has been made available to the community allowing for a full comparison between the various competing theoretical models and the experimental results. Following these recent developments, we have started the processes of filtering our theoretical dilepton spectra with the HADES filter. At this moment, final or full results are not yet available, we are only able to present a preliminary comparison of our spectral function approach to in-medium effects for one of the scenarios presented here for the $\mathrm{C}+\mathrm{C}$ reaction at $2 \mathrm{AGeV}$ : inmedium meson self-energies only receive contributions from excited nucleonic resonances only (the scenario depicted by blue curve in Fig. (屯). The comparison with the experimental data is shown in Fig. (5). A clear disagreement over almost the entire mass range is observed: at invariant masses below $0.7 \mathrm{GeV}$ the theoretical spectrum underpredicts data by a factor of 2 to 4 . This has been linked to a strong change of the $\Delta(1232)$ dilepton emission in this region. A comparison of the Brown-Rho/ collisional broadening scenario dilepton spectrum with the experimental data [27] 
only shows a discrepancy in the region around $0.6 \mathrm{GeV}$ invariant mass. The situation might be improved if additional contributions, which have previously been thought to contribute little, like $p n$ bremsstrahlung are included [28], an issue somewhat controversial due to a possible doublecounting problem. At masses above $0.7 \mathrm{GeV}$ the experimental spectrum is overestimated. This can however be cured by the inclusion of non-resonant contributions to in-medium meson self-energies, as these reduce the predicted dilepton cross-section in this mass region by an order of magnitude.

\section{Final conclusions}

We have presented results for the in-medium spectral functions of the $\rho$ and $\omega$ vector mesons. Interactions between vector mesons and nuclear matter were described using the eVMD model. Important modifications of meson properties with respect to vacuum are observed due to the excitation of nucleonic resonances in nuclear matter: the original Breit-Wigner peak gets broader and shifts towards higher masses. As second peak, due to excitation of nucleonic resonances, is observed at low invariant masses. A strong dependence on the meson three-momentum has also been noticed. Inclusion of additional in-medium contributions (non-resonant Compton scattering and $\sigma$ meson exchange contributions) results in a downward shift of the main mass branch and an additional increase of the collision width for the $\rho$. For the $\omega$ meson the results are more dramatic: the original peak dissolves while the secondary branch due to resonance excitations is narrow enough (30-100 MeV) to allow this meson to keep its quasi-particle properties in-medium.

Additionally, calculations for the dilepton emission spectra in $\mathrm{C}+\mathrm{C}$ heavy-ion reactions at 1.0 and 2.0 AGeV have been performed. A preliminary comparison with the published HADES data for $\mathrm{C}+\mathrm{C}$ collisions at $2 \mathrm{AGeV}$ reveals differences in the $0.2 \div 0.7$ mass range for the in-medium spectral function scenario. As suggested by other authors the discrepancy might be cured by the inclusion of reactions previously thought to be unimportant. For a final conclusion a comparison with the whole experimental results of the HADES collaboration (present and future) and of the DLS experiment is mandatory, for both invariant mass and transversal momentum spectra.

\section{References}

[1] G.E. Brown and M. Rho, Phys. Rev. Lett. 66, 2720 (1991); Phys. Rep. 269, 333 (1996).

[2] T. Hatsuda and S.H. Lee, Phys. Rev. C 46, R34 (1992); S. Leuopold, ibid. 64, 015202 (2001);

[3] C.M. Shakin and W.D. Sun, Phys. Rev. C 49, 1185 (1994); M. Asakawa and C.M. Rho, Phys. Rev. C 48, R526 (1993).

[4] M. Post, S. Leupold, and U. Mosel, Nucl. Phys. A 741, 81 (2004).

[5] D. Cabrera, E. Oset, and M.J. Vicente Vacas, Nucl. Phys. A 705, 90 (2002).

[6] F. Klingl, N. Kaiser, and W. Weise, Nucl. Phys. A 624, 527 (1997).

[7] K. Saito, K. Tsushima, and A.W. Thomas, arXiv:nucl-th/98110311.

[8] M.F.M. Lutz, G. Wolf, and B. Friman, Nucl. Phys. A 706, 431 (2002).

[9] P. Muehlich, V. Shklyar, S. Leupold, U. Mosel, and M. Post, Nucl. Phys. A 780, 187 (2006).

[10] R.J. Porter et al. [DLS Collaboration], Phys. Rev. Lett. 79, 1229 (1997). 
[11] W.K. Wilson et al. [DLS Collaboration], Phys. Rev. C 57 (1998) 1865.

[12] E.L. Bratkovskaya, W. Cassing, R. Rapp, and J. Wambach, Nucl. Phys. A 634, 168 (1998).

[13] C. Ernst, S.A. Bass, M. Belkacem, H. Stocker, and W. Greiner, Phys. Rev. C 58, 447 (1998);

[14] K. Shekter, C. Fuchs, A. Faessler, M. Krivoruchenko, and B. Martemyanov, Phys. Rev. C 68, 014904 (2003).

[15] R. Arnaldi et al. [NA60 Collaboration], Phys. Rev. Lett. 96 (2006) 162302.

[16] J. Friese, HADES Collaboration, Prog. Part. Nucl. Phys. 42, 235 (1999).

[17] G. Agakichiev et al., Phys. Rev. Lett. 98, 052302 (2007); W. Przygoda et al., Nucl. Phys. A 783, 583c (2007).

[18] A. Faessler, C. Fuchs, and M.I. Krivoruchenko, Phys. Rev. C 61, 035206 (2000).

[19] M.I. Krivoruchenko, B.V. Martemyanov, A. Faessler, and C. Fuchs, Ann. Phys. 296,

[20] A. Faessler, C. Fuchs, M.I. Krivoruchenko, and B.V. Martemianov, J. Phys. G 29, 603 (2003).

[21] M.D. Cozma, C. Fuchs, E. Santini, and A. Faessler, Phys. Lett. B 640, 170 (2006).

[22] M. Post and U. Mosel, Nucl. Phys. A 688, 808 (2001).

[23] A.I. Vainstein and V.I. Zakharov, Phys. Lett. B72, 368 (1978).

[24] S.J. Brodsky and G.R. Farrar, Phys. Rev. Lett. 31, 1153 (1973);

[25] C. Fuchs, M.I. Krivoruchenko, H. Yadav, A. Faessler, B.V. Martemyanov, and K. Shekther, Phys. Rev. C 67,025202 (2002).

[26] R. Machleidt, Phys. Rev. C 63, 024001 (2001).

[27] E. Santini, In-medium vector mesons and dileptons in heavy-ion collisions at 1-2 AGeV, talk given at the workshop "Electromagnetic probes for strongly interacting matter: the quest for medium modifications of hadrons", Trento, Italy (2007).

[28] E. Bratkovskaya, Dileptons from heavy-ion collisions: from SIS to FAIR and off-shell transport, talk given at the workshop "Electromagnetic probes for strongly interacting matter: the quest for medium modifications of hadrons", Trento, Italy (2007). 